'Becada en Capacitación Bases para el Manejo de la Patología Intestinal, Departamento de Gastroenterología, Clínica Las Condes, Santiago, Chile. ${ }^{2}$ Programa Enfermedad Inflamatoria Intestinal, Unidad de Coloproctología, Clínica Las Condes, Santiago, Chile. ${ }^{3}$ Programa Enfermedad Inflamatoria Intestinal, Servicio de Gastroenterología, Clínica Las Condes, Santiago, Chile. ${ }^{4}$ Departamento de Radiología, Clínica Las Condes, Santiago, Chile.

${ }^{5}$ Departamento de Anatomía Patológica, Clínica Las Condes, Santiago, Chile.

Conflicto de intereses: No existen conflictos de intereses. Fuente de apoyo financiero: No se recibió ayuda financiera.

Recibido el 1 de junio de 2016, aceptado el 6 de septiembre de 2016.

Correspondencia a: Dr. Rodrigo Quera Clínica Las Condes, Lo Fontecilla 441, Las Condes, Santiago, Chile. Teléfono: (56) (2) 26108048 rquera@clc.c

\section{Mesotelioma benigno como causa de dolor abdominal inespecífico en un paciente con Enfermedad de Crohn}

\author{
DANIELA FLUXÁ ${ }^{1}$, UDO KRONBERG ${ }^{2}$, JAIME LUBASCHER ${ }^{3}$, \\ ANDRÉS O'BRIEN ${ }^{4}$, FACUNDO LAS HERAS ${ }^{5}$, \\ PATRICIO IBÁÑEZ ${ }^{3}$, RODRIGO QUERA ${ }^{3}$
}

\section{Benign multicystic peritoneal mesothelioma in a patient with Crohn disease}

Benign multicystic peritoneal mesothelioma is an uncommon lesion arising from the peritoneal mesothelium. It is asymptomatic or presents with unspecific symptoms. Imaging techniques may reveal it, however the final diagnosis can only be made by histopathology. Surgery is the only effective treatment considering its high recurrence rate. We report a 19 years old male with Crohn's disease. Due to persistent abdominal pain, an abdominal magnetic resonance imaging was performed, showing a complex cystic mass in the lower abdomen. The patient underwent surgery and the lesion was completely resected. The pathological study reported a benign multicystic peritoneal mesothelioma.

(Rev Med Chile 2016; 144: 1612-1616)

Key words: Crohn's disease; Cystic; Mesothelioma; Parietal Peritoneum; Peritoneum.
$\mathrm{E}$ mesotelioma multiquístico peritoneal benigno (MMPB), también conocido como quiste de inclusión, es una lesión infrecuente que se origina de las células mesoteliales del peritoneo $^{1}$ existiendo hasta la fecha menos de 200 casos publicados ${ }^{2}$. Su patogénesis aún es incierta y controversial ${ }^{3,4}$ siendo su diagnóstico preoperatorio complejo ${ }^{3}$. El MMPB se presenta con mayor frecuencia en mujeres en edad reproductiva ${ }^{1,3,5} \mathrm{y} \mathrm{a}$ diferencia del mesotelioma maligno no está asociado a la exposición de asbestos ${ }^{5}$. A la fecha sólo existe un caso reportado con respecto a reacción mesotelial en un paciente con Enfermedad de Crohn $(E C)^{6}$. Presentamos el caso de un paciente con EC que durante su evolución desarrollo un MMPB. Dado que aún no existe conocimiento sobre la relación entre ambas enfermedades nos parece importante reportar esta asociación, ya que podría contribuir al diagnóstico y manejo de futuros casos.

\section{Caso clínico}

Paciente de sexo masculino de 19 años de edad, con antecedentes de una fístula perianal tratada con antibióticos, consulta por cuadro digestivo de 5 meses de evolución caracterizado por dolor abdominal difuso tipo cólico y deposiciones de consistencia variable, explosivas, sin sangre. En exámenes de laboratorio destaca hemoglobina (Hb) 11,3, velocidad horaria de sedimentación (VHS) $33 \mathrm{~mm} / \mathrm{h}$ y proteína $\mathrm{C}$ reactiva (PCR) $4,16 \mathrm{mg} / \mathrm{L}$ (valor normal $<1$ ). Se decide realizar estudio colonoscópico que reveló úlceras en íleon con biopsias compatibles con el diagnóstico de EC, iniciándose budesonida por 3 meses y azatioprina (AZA) $125 \mathrm{mg} /$ día $(2 \mathrm{mg} / \mathrm{Kg})$. Tras 6 meses de uso de AZA, el paciente persistió con anemia, PCR 35 $\mathrm{mg} / \mathrm{L}$ (valor normal $<10$ ) y calprotectina fecal elevada (semi cuantitativa $>200 \mathrm{ug} / \mathrm{g}$ ) iniciándose tratamiento con adalimumab. Luego de 15 meses 
de tratamiento con terapia biológica consulta por cuadro de 10 días de evolución caracterizado por dolor hipogástrico, distensión abdominal y dolor pre y post miccional. Dentro de los exámenes de laboratorio destaca VHS y PCR levemente elevadas $(28 \mathrm{~mm} / \mathrm{h}$ y $37 \mathrm{mg} / \mathrm{L}$ (valor normal $<10)$ respectivamente). Inicialmente recibió tratamiento sintomático, pero dado persistencia de sus síntomas se solicitó una resonancia magnética (RM) de abdomen-pelvis que mostró compromiso multifocal del íleon terminal asociado a pequeñas linfoadenopatías ileocólicas y una compleja masa hipogástrica de aspecto quístico, multilocular, de septos gruesos que realza post contraste (Figura 1A). Se consideró un MMPB dentro del diagnóstico diferencial y el paciente fue sometido a cirugía. Durante el procedimiento, una vez expuesta la cavidad peritoneal, se observaron numerosos quistes de paredes delgadas y contenido citrino, intensamente adheridos al peritoneo pélvico, vejiga y colon sigmoides (Figura 1B). Uno de los quistes fue resecado y enviado a biopsia rápida, informándose como mesotelioma benigno. Macroscópicamente el apéndice y el colon derecho no presentaban alteraciones, mientras que el mesenterio adyacente a los últimos $40 \mathrm{~cm}$ del íleon terminal se observaba engrosado. Se decidió realizar una colonoscopia intraoperatoria la cual mostró úlceras en los últimos $30 \mathrm{~cm}$ de íleon y una estenosis franqueable a este nivel. La lesión quística fue cuidadosamente resecada de forma completa, se liberó el mesenterio del íleon terminal y se realizó una resección ileocólica con anastomosis latero-lateral y sutura manual. La biopsia diferida informó un quiste de inclusión peritoneal multilocular benigno con metaplasia escamosa y expresión de calretinina y citoqueratina (Figura 2). El íleon terminal, colon ascendente y
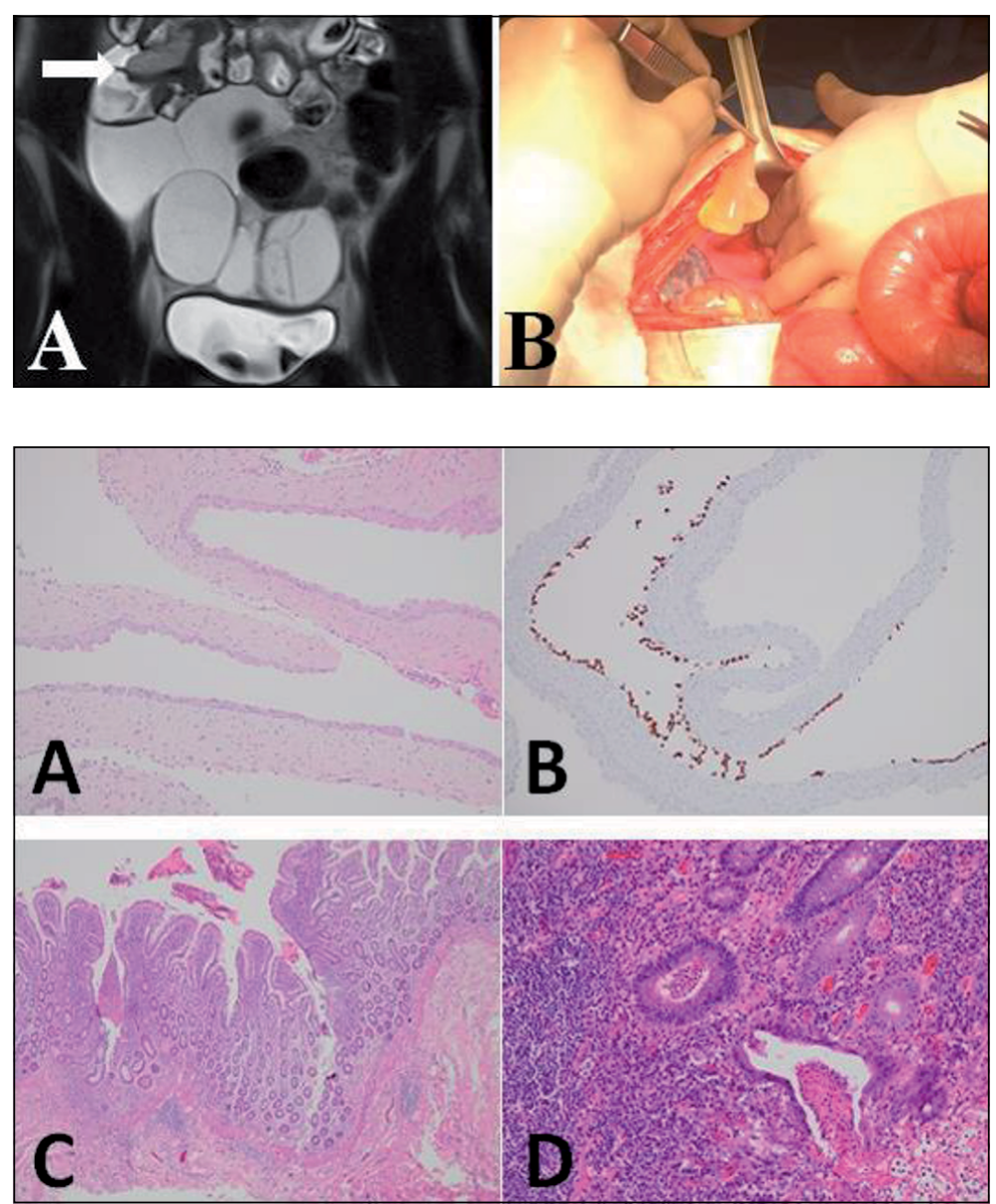

Figura 1. Hallazgos radiológicos (A) y quirúrgicos (B). Corte coronal secuencia T2 de alta resolución (A) en donde se observa una masa pélvica multiquística adyacente al íleon terminal, el cual se encuentra engrosado (flecha), con lóculos de señal alta e intermedia que representan contenido seroso y hemorrágico, respectivamente. (B) Numerosos quistes de paredes delgadas y el contenido citrino, intensamente adheridos al peritoneo pélvico.

Figura 2. Hallazgos histológicos relevantes. A: Quiste benigno compuesto de tejido fibroconectivo con revestimiento mesotelial prominente (H/E 100X). B: Inmunotinción para calretinina que destaca el revestimiento mesotelial (Calretinina 100X). C: Ileítis crónica activa con ulceración superficial y compromiso inflamatorio de toda la pared intestinal (H/E 40x). D: Criptitis e inflamación intraepitelial prominente (H/E 200X). Los hallazgos descritos en $\mathbf{C}$ y $\mathbf{D}$ apoyan el diagnóstico de Enfermedad de Crohn. 
apéndice mostraron ileitis crónica activa ulcerada, con microabscesos crípticos y reacción transmural granulomatosa, consistente con EC activa y mucosa colónica con inflamación crónica inespecífica. El postoperatorio inmediato se caracterizó por la presencia de íleo adinámico el cual se resolvió a los dos días, siendo el paciente dado de alta sin otras complicaciones. Se indicó mantener terapia biológica, sin embargo, el paciente abandonó el seguimiento y reconsultó a los seis meses por dolor abdominal, diarrea y dolor perianal. Durante este período de tiempo el paciente no continuó la terapia sugerida y sólo se mantuvo con AZA en dosis subterapéuticas ( $50 \mathrm{mg} /$ día). Se solicitó una RM de pelvis que mostró un absceso y fistula perianal compleja decidiéndose su hospitalización para drenaje e instalación de sedal, iniciando posteriormente terapia con AZA $2 \mathrm{mg} / \mathrm{kg} / \mathrm{día}$ e infliximab $5 \mathrm{mg} / \mathrm{kg}$ en esquema de inducción $(0,2$ y 6 semanas) y luego cada 8 semanas (esquema de mantención).

\section{Discusión}

Hemos descrito el caso de un paciente de 19 años con EC quien desarrolló un MMPB a los 21 meses post diagnóstico de su Enfermedad Inflamatoria Intestinal (EII).

El MMPB es una lesión infrecuente, caracterizado por quistes multiloculados, múltiples o solitarios, de paredes delgadas de contenido seroso o hemático ${ }^{7}$. Aunque su patogénesis aún se desconoce, se han propuesto cinco hipótesis con respecto a su origen: inflamatorio, neoplásico, genético, hormonal y embriológico ${ }^{5}$. La hipótesis inflamatoria postula que el origen de este tumor estaría en directa relación con inflamación crónica peritoneal, historia de cirugías previas o enfermedad inflamatoria pélvica ${ }^{1,5,8}$.

El diagnóstico preoperatorio es complejo ${ }^{3}$ y requiere de un alto índice de sospecha ${ }^{9}$. En la mayoría de los casos el MMPB no produce síntomas y puede adherirse a órganos vecinos ${ }^{1}$. Ocasionalmente puede presentarse como dolor abdominal/ pélvico crónico o intermitente, náuseas, vómitos, distensión abdominal o masa abdominal/pélvi$\mathrm{ca}^{2,3,10}$. Las técnicas de imágenes disponibles tales como la ecografía, tomografía computada y RM son capaces de demostrar la lesión ${ }^{1}$. En la ecografía el MMPB puede apreciarse como una masa multiquística, con vascularización de los septos, sin calificaciones. En la tomografía computada puede observarse una masa multiquística con lóbulos de diferente densidad mientras que en la RM se aprecia una lesión multiquística con áreas de intensidad baja-intermedia en T1 e intensidad intermedia-alta en $\mathrm{T} 2^{5}$. No obstante, ninguna de las imágenes previamente señaladas es capaz de entregar un diagnóstico definitivo entre MMPB y otras lesiones multiloculares quísticas ${ }^{5,11}$. En relación a lesiones benignas, los diagnósticos diferenciales más importantes incluyen el linfangioma quístico o tumor adenomatoide, mientras que el diagnóstico diferencial con respecto a lesiones malignas incluye al mesotelioma maligno $y$ tumores serosos que invaden el peritoneo ${ }^{1,4}$. Finalmente, el diagnóstico definitivo sólo se puede determinar a través de la histopatología ${ }^{3,9}$, donde puede observarse metaplasia adenomatoidea focal o escamosa ${ }^{7}$. El uso de tinciones de inmunohistoquímica específicas para células mesoteliales, como la calretinina y citoqueratina $5 / 6$, permite la distinción entre MMPB y otras entidades ${ }^{3,5}$. Ambas tinciones fueron positivas en nuestro paciente.

La cirugía ha mostrado ser el único tratamiento efectivo para el MMPB y consiste en la resección completa de la lesión quística ${ }^{1,3,9}$. Aunque el pronóstico es favorable, la recurrencia puede alcanzar entre el $27-75 \%{ }^{3-5,10}$, probablemente por la persistencia del factor causante original ${ }^{5}$. Esta evolución, plantea la necesidad de un seguimiento sistemático prolongado, probablemente de por vida $^{3,5,9}$. En ese sentido, nuestro paciente no sólo necesitará un seguimiento cercano, sino deberá tener un tratamiento óptimo de su EC, la cual, en teoría, podría haber jugado un rol en el desarrollo o evolución del MMPB. Por otro lado, se han descrito escasos reportes de transformación maligna $^{12,13}$. Con respecto a lo anterior, algunos cirujanos han propuesto un manejo quirúrgico más agresivo seguido de quimioterapia ${ }^{10,14}$. Sin embargo, la potencial utilidad de la quimioterapia adyuvante y radioterapia es incierta dado las características principalmente benignas del $\mathrm{MMPB}^{5}$. Otros también han descrito la posibilidad que un mesotelioma maligno simule una enfermedad inflamatoria intestinal ${ }^{15}$.

Aunque existen varias publicaciones sobre mesotelioma peritoneal, dos de ellas nacionales ${ }^{16,17}$, solamente un artículo ha descrito la presencia de reacción mesotelial en la pieza quirúrgica de 
un paciente con EC de larga evolución ${ }^{6}$. En ese caso, el paciente tenía una historia de 20 años de síntomas posiblemente relacionados a su EC, sin recibir ningún tratamiento durante ese período, perpetuándose así el proceso inflamatorio. Se cree que los efectos locales de las citoquinas liberadas a causa de los cambios inflamatorios en las capas más externas de la pared intestinal son las que provocan anormalidades en el tejido conectivo de la serosa ${ }^{18}$. Nuestro caso es diferente, ya que el paciente recibió terapia con AZA y adalimumab $y$ en menos de dos años desarrollo un MMPB. A pesar del corto período de evolución, la inflamación crónica podría haber jugado un rol, ya que al realizar la colonoscopia intraoperatoria, y a pesar del uso de terapia combinada, el paciente aún presentaba signos de actividad en los últimos 30 $\mathrm{cm}$ de íleon. Aunque otros factores podrían estar involucrados en el desarrollo del $\mathrm{MMPB}^{5}$, una revisión reciente plantea el rol del mesenterio en la patogénesis y evolución de la $\mathrm{EC}^{19}$. Además, otros han publicado la presencia de quistes de inclusión peritoneal en pacientes con EC los cuales fueron diagnosticados por $\mathrm{RM}^{20}$.

En conclusión, el MMPB es una lesión infrecuente que podría estar relacionada con la presencia de inflamación crónica. Aunque se ha considerado una lesión benigna, presenta una alta tasa de recurrencia y por lo tanto, la cirugía sigue siendo el tratamiento de primera línea con seguimiento prolongado en el tiempo. Menos de 200 casos han sido publicados en la literatura, y sólo existe un caso respecto a reacción mesotelial en un paciente con EC. Es posible que la inflamación crónica transmural relacionada a la EC tenga un rol en el desarrollo y/o evolución del MMPB. Estudios con un mayor número de casos podría dilucidar si estas dos enfermedades están realmente asociadas.

\section{Referencias}

1. Safioleas MC, Constantinos K, Michael S, Konstantinos G, Constantinos S, Alkiviadis K. Benign multicystic peritoneal mesothelioma: a case report and review of the literature. World J Gastroenterol 2006; 12 (35): 5739-42.

2. Iacoponi S, Calleja J, Hernández G, de la Cuesta RS. Asymptomatic peritoneal carcinomatosis originating from benign cystic peritoneal mesothelioma. Ecancermedicalscience 2015; 9: 605 .
3. Wang TB, Dai WG, Liu DW, Shi HP, Dong WG. Diagnosis and treatment of benign multicystic peritoneal mesothelioma. World J Gastroenterol 2013; 19 (39): 6689-92.

4. Dzieniecka M, Kałużyński A. Benign multicystic peritoneal mesothelioma (BMPM) - case report and review of the literature. Pol J Pathol 2011; 62 (2): 122-4.

5. Cavallaro A, Berretta M, Lo Menzo E, Cavallaro V, Zanghì $\mathrm{A}$, Di Vita M, et al. Cystic peritoneal mesothelioma: report of a case. Surg Today 2011; 41 (1): 141-6.

6. Wilkinson L, De P, Bloxham C. Mesothelial reaction in longstanding Crohn's ileitis simulating papillary mesothelioma. J Clin Pathol 2008; 61 (10): 1119-21.

7. Tangjitgamol S, Erlichman J, Northrup H, Malpica A, Wang X, Lee E, et al. Benign multicystic peritoneal mesothelioma: cases reports in the family with diverticulosis and literature review. Int J Gynecol Cancer; 15 (6): 1101-7.

8. Villaschi S, Autelitano F, Santeusanio G, Balistreri P. Cystic mesothelioma of the peritoneum. A report of three cases. Am J Clin Pathol 1990; 94 (6): 758-61.

9. Somasundaram S, Khajanchi M, Vaja T, Jajoo B, Dey AK. Benign multicystic peritoneal mesothelioma: a rare tumour of the abdomen. Case Rep Surg 2015; 2015: 613148.

10. Momeni M, Pereira E, Grigoryan G, Zakashansky K. Multicystic benign cystic mesothelioma presenting as a pelvic mass. Case Rep Obstet Gynecol 2014; 2014: 852583.

11. Yang DM, Jung DH, Kim H, Kang JH, Kim SH, Kim $\mathrm{JH}$, et al. Retroperitoneal cystic masses: CT, clinical, and pathologic findings and literature review. Radiographics 2004; 24 (5): 1353-65.

12. González-Moreno S, Yan H, Alcorn KW, Sugarbaker $\mathrm{PH}$. Malignant transformation of "benign" cystic mesothelioma of the peritoneum. J Surg Oncol 2002; 79 (4): 243-51.

13. Hejmadi R, Ganesan R, Kamal NG. Malignant transformation of a well-differentiated peritoneal papillary mesothelioma. Acta Cytol 2003; 47 (3): 517-8.

14. Elbouhaddouti H, Bouassria A, Mouaqit O, Benjelloun el B, Ousadden A, Mazaz K, et al. Benign cystic mesothelioma of the peritoneum: a case report and literature review. World J Emerg Surg 2013; 8 (1): 43.

15. Oberto C, Schwarz KB, Zambidis E, Campbell AB, Paidas C, Lindyberg K, et al. Malignant peritoneal mesothelioma in a pediatric patient mimicking inflammatory bowel disease. Dig Dis Sci 2004; 49 (3): 434-7.

16. Bannura G, Contreras J, Peñaloza P. Quiste mesotelial simple gigante abdomino-pélvico. Rev Chil Cir 2008; 60 (1): 67-70. 
17. Saelzai E, Vela H, Cortés F, Alarcón T. Mesotelioma quístico peritoneal. Rev Chil Pediatr 1987; 58 (2): 15760.

18. Borley NR, Mortensen NJ, Jewell DP, Warren BF. The relationship between inflammatory and serosal connective tissue changes in ileal Crohn's disease: evidence for a possible causative link. J Pathol 2000; 190 (2): 196-202.

19. Li Y, Zhu W, Zuo L, Shen B. The role of the mesentery in Crohn's disease: The contributions of nerves, vessels, lymphatics, and fat to the pathogenesis and disease course. Inflamm Bowel Dis 2016; 22 (6): 1483-95.

20. Mazziotti S, D’Angelo T, Racchiusa S, Salamone I, Blandino A, Ascenti G. Peritoneal inclusion cysts in patients affected by Crohn's disease: magnetic resonance enterography findings in a case series. Clin Imaging 2016; 40 (1): 152-5. 